\title{
THE NEUTRAL ENVELOPES AROUND AGB AND POST-AGB OBJECTS
}

\author{
Their Structure and Kinematics
}

\author{
H. OLOFSSON \\ Stockholm Observatory \\ S-133 36 Saltsjöbaden, Sweden
}

\begin{abstract}
This review discusses the large-scale geometry and kinematics of envelopes around evolved stars, as inferred from radio line observations of circumstellar molecules, as a function of the evolutionary stage of the central object. In particular, the drastic change in morphology from largely spherical envelopes around asymptotic giant branch stars to distinctly non-spherical neutral envelopes around planetary nebulae is addressed. In addition, the small-scale structure of the envelopes, i.e., whether the circumstellar medium is smooth or clumpy, is discussed. Finally, a scenario for the morphological evolution, based on the assumption of a highly clumped medium, is presented.
\end{abstract}

\section{Introduction}

It is nowadays well established that stars on the Asymptotic Giant Branch (AGB) eventually lose considerable amounts of matter in a stellar wind, and that it is this process that significantly limits their lifetime on the AGB. The wind is slow enough that the envelope of gas and dust, formed by the mass loss, will remain in the vicinity as the star leaves the AGB and evolves towards its final stage as a white dwarf. Thus, the morphology and kinematics of the circumstellar envelopes (CSEs) provide us with information on the temporal evolution of the stellar mass loss on, as well as beyond, the AGB. The CSE also plays an important role in the formation of the planetary nebula $(\mathrm{PN})$. The small-scale structure of the circumstellar medium gives information on the details of the mass-loss process. This is of crucial importance to our understanding of the mass-loss mechanism, which appears to involve physical processes that are critically dependent 
on density, temperature, etc. Due to lack of space, only the most recent, relevant publications are referred to.

\section{The Probes}

The small- and large-scale structure of the circumstellar medium can be probed by dust continuum emission and in atomic and molecular lines. Both types of probes have their advantages and disadvantages, and we will here concentrate on the molecular radio lines. In general, line emission has the advantage that it also carries kinematic information.

The present number of molecular species detected at radio wavelengths in AGB or post-AGB CSEs is 49 (Olofsson 1997). The majority of these are detected towards only one object (IRC+10216), or at most a few objects. Good statistics can be obtained essentially only from four molecules: $\mathrm{SiO}$ ( $\geq 450$ objects), $\mathrm{OH}$ ( $\geq 2000$ objects), $\mathrm{H}_{2} \mathrm{O}$ ( $\geq 300$ objects), and $\mathrm{CO}(\geq 450$ objects) [compilations of data can be found in Benson et al. (1990) and Loup et al. (1993)]. The first three, which are only seen in maser emission (except for $\mathrm{SiO}$ where there are also "thermal" lines), are restricted to oxygen-rich envelopes (i.e. $\mathrm{C} / \mathrm{O}<1, \mathrm{O}-\mathrm{CSEs}$ ), but may be used to study the entire mass-loss phase of the AGB evolution, and to some extent also the post-AGB evolution (except for $\mathrm{SiO}$ ). The $\mathrm{CO}$ emission is "thermal" in nature and probes both $\mathrm{O}-\mathrm{CSEs}$ and $\mathrm{C}-\mathrm{CSEs}$ (i.e. those with $\mathrm{C} / \mathrm{O}>$ 1) in the AGB-phase and beyond, but the observational space is limited to about $10 \mathrm{kpc}$ (Olofsson 1989). The masers are in general stronger, and in the LMC six objects have been detected in $\mathrm{OH}(1612 \mathrm{MHz}$ ) emission (Wood et al. 1992) and one object in $\operatorname{SiO}(v=1, J=2 \rightarrow 1$ ) emission (van Loon et al. 1996).

\section{The Deconvolution Problem}

In the best case the observational data consist of two-dimensional brightness distributions at different line-of-sight velocities in a transition of a species $\mathrm{X}, I\left(\alpha, \delta, v_{\mathrm{z}}, \mathrm{X}(u \rightarrow l)\right)$. In order to get from this to the density distribution of species $\mathrm{X}, \rho_{\mathrm{X}}(r, \theta, \varphi)$, and to the kinematics for the CSE in the region probed by $\mathrm{X}(u \rightarrow l), v_{\mathrm{e}, \mathrm{X}}(r, \theta, \varphi)$, one has to correct for beam smearing (a severe problem since most emissions are unresolved by single-dish observations) and line-of-sight contamination (the $v_{\mathbf{z}}$ information helps if the kinematics are simple), and finally calculate the excitation and the radiative transfer. For the latter a knowledge of the detailed density structure (i.e., a smooth or clumpy medium) as well as of the radiation field (which depends strongly on the evolutionary stage of the central star) is required. The density distribution $\rho_{\mathrm{X}}$ is determined by the chemistry, i.e., one has to identify whether species $\mathrm{X}$ is of photospheric origin or if it is a product of processes 
in the CSE (see, e.g., Nercessian et al. 1989; Cherchneff \& Glassgold 1993; Millar \& Herbst 1994). In the latter case the molecule is either a direct photodissociation product or else it is produced in a photo-induced chemistry, and a knowledge of the relevant radiation fields (interstellar UV field, chromospheric radiation, hot central star radiation in the case of post-AGB objects) is required. It is the excitation and the chemistry that determine the region probed by the $\mathrm{X}(u \rightarrow l)$ line. Observations of several species and transitions will eventually give the true total density distribution $\rho(r, \theta, \varphi)$ and the true kinematics $v_{\mathrm{e}}(r, \theta, \varphi)$. The mass-loss geometry and history are finally obtained from the continuity equation, $\dot{M}\left(t-t_{\text {ret }}, \theta, \varphi\right)=4 \pi r^{2} \rho v_{\mathrm{e}}$ [where $t_{\text {ret }}$ is determined by $r$ and $v_{\mathrm{e}}(r)$ ], provided that a number of conditions are fulfilled.

\section{Morphology and Kinematics}

\subsection{AGB-CSEs}

As outlined in the previous section, different probes probe different regions. Although this has obvious advantages, it also means that the connection between density structures at different scales is usually not easy to obtain. At the smallest scales we are restricted to maser emission, and unfortunately there are no useful probes for C-CSEs [two objects have been mapped in HCN maser emission, but there is no resolved structure (Carlstrom et al. 1990; Lucas \& Guilloteau 1992)]: $\mathrm{SiO}(43 \mathrm{GHz}$ ) and $\mathrm{H}_{2} \mathrm{O}(22 \mathrm{GHz}$, low $-\dot{M} \mathrm{CSEs})$ at $\leq 10^{14} \mathrm{~cm}, \mathrm{H}_{2} \mathrm{O}(22 \mathrm{GHz}$, higher $-\dot{M}$ CSEs $)$ and $\mathrm{OH}(1665 / 1667 \mathrm{MHz})$ at $\leq 10^{15} \mathrm{~cm}$. It is for various reasons very difficult to draw any definite conclusions on the geometry and kinematics of CSEs from the maser data. The most recent $\mathrm{SiO}$ observations show roughly circular ring structures (Diamond et al. 1994; Miyoshi et al. 1994) or elongated structures (Colomer et al. 1996). Important to note here is that at these small scales $\left(\leq 0{ }^{\prime \prime} 1\right)$ the position of the star has to be assumed. In only one case has the position and size of the star been measured simultaneously with the maser emission $\left[\mathrm{H}_{2} \mathrm{O}(22 \mathrm{GHz})\right.$ towards $\mathrm{W}$ Hya], and the result is an apparently spherical shell of emitting gas centred on the star (Reid \& Menten 1990). In many other cases the $\mathrm{H}_{2} \mathrm{O}$ brightness distributions are highly asymmetric (see, e.g., Bowers \& Johnston 1994; Yates \& Cohen $1994)$. Also at the sligthly larger scales probed by the $\mathrm{OH}(1665 / 1667 \mathrm{MHz})$ masers it is not clear whether the density distribution is largely spherical, or if for instance there exists equatorial density enhancements (Bowers et al. 1989; Chapman et al. 1991, 1994). Kinematically it appears that a fair fraction of the gas has reached the terminal expansion velocity (as measured at large scales) already at about $10^{15} \mathrm{~cm}$ (Bowers \& Johnston 1994).

At the larger scales, $\geq 10^{16} \mathrm{~cm}$, the situation is better, from both a the- 
oretical and an observational point of view. Here the $\mathrm{OH}(1612 \mathrm{MHz})$ maser emission outlines more or less overall spherically symmetric shells in about 15 cases (see e.g. Herman et al. 1985; Welty et al. 1987). The best examples are $\mathrm{OH} 26.5+0.6$ and $\mathrm{OH} 127.8-0.0$ (which may be a supergiant) (Bowers \& Johnston 1990). The evidence for sphericity is further strengthened by good fits to the size $-v_{\mathrm{z}}$ relation, $\theta\left(v_{\mathrm{z}}\right)=\theta(0)\left[1-\left(v_{\mathrm{z}} / v_{\mathrm{e}}\right)^{2}\right]^{1 / 2}$, where $\theta\left(v_{\mathrm{z}}\right)$ is the measured size of the emission in a narrow velocity range around $v_{\mathrm{z}}$, and $\theta(0)$ is the source size at the systemic velocity, expected to apply for a spherical shell expanding at the constant velocity $v_{\mathrm{e}}$. At roughly the same size scale, maps of "thermal" $\mathrm{SiO}$ emission towards 10 objects show geometries consistent with overall spherical symmetry (Lucas et al. 1992; Sahai \& Bieging 1993). At slightly larger scales, $\geq 5 \times 10^{16} \mathrm{~cm}$, about 25 objects have now been mapped in various $\mathrm{CO}$ transitions using single telescopes, but since the majority of the objects are just barely resolved, and the dynamic range in the maps is limited, one should not put too much faith in the reported high frequency of elongations and asymmetries in the brightness distributions (Bujarrabal \& Alcolea 1991; Stanek et al. 1995). In the four cases where the emission is clearly spatially resolved at scales of $10^{17}-10^{18} \mathrm{~cm}$, namely the C stars IRC +10216 (Truong-Bach et al. 1991), U Ant, S Sct, and TT Cyg (Olofsson et al. 1996), there is good evidence for overall spherical symmetry and isotropic expansion at a constant velocity.

Only in IRC +10216 has emission from a fair number of molecular species (16) been observed at high angular resolution (see, e.g., Bieging \& Tafalla 1993; Guélin et al. 1993; Dayal \& Bieging 1995; Gensheimer et al. 1995; Guélin et al. 1997). The combined picture is a CSE with an overall spherical symmetry (however, see below). Recent observations of six species towards the carbon star CIT 6, on the other hand, point to a marked elongation at $\mathrm{PA} \approx 155^{\circ}$ (Lindqvist et al. 1998).

Departures from "sphericity" certainly exist. A faster, bipolar wind and a (probably) largely spherical AGB-CSE (perhaps open at the poles) give a good explanation for the CO data on $o$ Ceti (Planesas et al. 1990), X Her (Kahane \& Jura 1996), V Hya (Kahane et al. 1996), and $\pi^{1}$ Gru (Sahai 1992). Both the velocities of the AGB-CSEs (3-12 $\mathrm{km} \mathrm{s}^{-1}$ ) and the bipolar winds $\left[(3-25) / \cos i \mathrm{~km} \mathrm{~s}^{-1}\right.$, where $i$ is the angle between the bipolar outflow axis and the line of sight) are fairly small in these cases (except for V Hya; see also Knapp et al. 1997). R Leo appears to have (possibly) a bipolar outflow $\left(\sim 3 / \cos i \mathrm{~km} \mathrm{~s}^{-1}\right)$ close to the star $\left(\leq 2 \times 10^{14} \mathrm{~cm}\right)$ (Cernicharo et al. 1994). There is a symmetry axis in the IRC $+10216 \mathrm{CSE}$ in the sense that the brightness distributions appear open at $\mathrm{PA} \approx 20^{\circ}$ (see, e.g., Lucas et al. 1995). Furthermore, the star is not at the center of the extended envelope, possibly suggesting a binary nature for IRC +10216 (Guélin et al. 1993). The CSE of V Cyg has been convincingly shown to be elongated 
and not centered on the star (Bujarrabal \& Alcolea 1991). Finally, in some stars there is evidence for low-intensity, higher-velocity wings (Bujarrabal \& Alcolea 1991).

The data on IRC +10216 also show how difficult it is to infer the density structure from a single line. The $\mathrm{SiC}_{2}\left(4_{23} \rightarrow 3_{22}\right)$ line shows an opening in the CSE at $\mathrm{PA} \approx 20^{\circ}$ as do many other lines (Lucas et al. 1995), while both the $\mathrm{SiC}_{2}\left(4_{04} \rightarrow 3_{03}\right)$ (Takano et al. 1992) and the $\mathrm{SiC}_{2}\left(4_{22} \rightarrow 3_{21}\right.$ ) (Gensheimer et al. 1995) lines have their emission maxima in this direction.

\subsection{EARLY POST-AGB CSEs}

The early post-AGB objects can be crudely divided into three classes when it comes to the morphology and kinematics of the AGB-CSEs. It is not clear that these groups outline an evolutionary sequence for post-AGB objects, or whether the apparent differences are due to inherent differences (e.g. binarity). In general, the classification of post-AGB objects is a tricky business, and, in fact, many objects so classified may not even be post-AGB objects.

In the first class we have CRL 618 and CRL 2688, objects surrounded by large, essentially unperturbed and overall spherically symmetric AGBCSEs that expand with a velocity of $\sim 20 \mathrm{~km} \mathrm{~s}^{-1}$ (Truong-Bach et al. 1990; Phillips et al. 1992; Yamamura et al. 1996). However, there is a distinct difference between the $\mathrm{CO}$ line profiles of these objects and those of the CSEs of AGB objects. The presence of low-intensity, high-velocity wings in the former suggests very high-velocity outflows [CRL 618, 200/cos $i \approx$ $280 \mathrm{~km} \mathrm{~s}^{-1}$, Gammie et al. (1989), Cernicharo et al. (1989); CRL 2688, $\sim 40 / \cos i$ and 100/cos $i \mathrm{~km} \mathrm{~s}^{-1}$, Jaminet et al. (1992), Young et al. (1992)]. The bipolar nature of these high-velocity winds is now established (Neri et al. 1992; Yamamura et al. 1995; Bieging \& Nguyen-Q-Rieu 1996), and there is good evidence for elongated density enhancements at small scales (Nguyen-Q-Rieu et al. 1986; Hajian et al. 1995; Martin-Pintado et al. 1995). In CRL 2688 the HCN emission provides evidence for rotation in the molecular gas in the direction orthogonal to the bipolar axis (Bieging \& Nguyen-Q-Rieu 1996).

In the second class the line profiles are dominated by the high-velocity outflow, and there seems to be little trace of a normal AGB-CSE, i.e. a fair fraction of the AGB-CSE gas has very likely been accelerated to high velocities. Examples of this class are M1-92 and $\mathrm{OH} 231.8+4.2$. In the former, some gas remains in the vicinity of the star, but an elongated cavity has developed and the polar gas expands at $\sim 50 / \cos i \approx 65 \mathrm{~km} \mathrm{~s}^{-1}$ (Bujarrabal et al. 1994). In the latter, the dominating $\mathrm{CO}$ emission comes from a very high-velocity $\left(\sim 250 / \cos i \approx 330 \mathrm{~km} \mathrm{~s}^{-1}\right)$, bipolar outflow that 
follows the optical outline (Alcolea et al. 1996).

In the third class high-velocity winds also exist, but spectra obtained towards the star are dominated by a strong, narrow $\left(5-10 \mathrm{~km} \mathrm{~s}^{-1}\right)$ feature, possibly indicating the presence of a strong equatorial density enhancement. Examples of this class are IC 2220 (Nyman et al. 1993) and the Red Rectangle (Jura et al. 1995).

Finally, we mention the CO map data on an object whose post-AGB nature is not conclusively established, $89 \mathrm{Her}$. There appear to be two CSEs, an inner envelope and an outer shell as inferred from $\mathrm{CO}(1 \rightarrow 0)$ data, with geometries and kinematics that are not easily interpreted (Alcolea \& Bujarrabal 1995). On the other hand, when integrated over the entire velocity range of the emission, the brightness distribution is circularly symmetric.

It should be noted here that the external parts of thick AGB-CSEs may become very cold because of adiabatic cooling during the expansion (Sahai 1990), and hence the gas may escape detection. For the high-velocity gas the cooling may become even more severe, and the gas may in fact only be detectable in absorption against the microwave background.

\subsection{LATE POST-AGB CSEs}

There are presently about $45 \mathrm{PNe}$ detected in $\mathrm{CO}$ emission (Huggins et al. 1996), and a fair fraction of these have been mapped in some detail. Only in the youngest $\mathrm{PNe}$ does one see an essentially unperturbed AGB-envelope. The best example is provided by NGC 7027 which is surrounded by a large AGB-CSE that appears overall spherically symmetric and that expands with $\sim 15 \mathrm{~km} \mathrm{~s}^{-1}$ (Bieging et al. 1991). At smaller scales there is a cavity, and the $\mathrm{CO}$ data suggest that it is surrounded by a prominent toroidal density distribution (Graham et al. 1993).

For the more evolved PNe the CO maps show brightness distributions that to a large extent follow the optical morphology, e.g. hour-glass structures (NGC 2346, Bachiller et al. 1989b) or rings (NGC 6720, Bachiller et al. 1989a; NGC 6781, Bachiller et al. 1993). In all cases this suggests equatorial density enhancements (probably of different strengths). The dominating $\mathrm{CO}$ emission has expansion velocities that lie at the high end of what is observed for AGB-CSEs $\left(\geq 20 \mathrm{~km} \mathrm{~s}^{-1}\right)$, probably indicating that some acceleration of the gas has taken place. An extreme example is $\mathrm{BD}+30^{\circ} 3639$ where the gas expands with $\sim 50 \mathrm{~km} \mathrm{~s}^{-1}$ (Bachiller et al. 1991). Bipolar winds are present but the expansion velocities are quite moderate $(\sim 30 / \cos i$ $\mathrm{km} \mathrm{s}^{-1}$ ), i.e. much lower than for the early post-AGB CSEs, and they become significantly rarer among the elliptical PNe (Jaminet et al. 1991; Sahai et al. 1991; Sahai et al. 1994). 


\section{Small-Scale Structure of the Circumstellar Medium}

It has traditionally been assumed that the mass loss occurs in the form of a steady and smooth stellar wind, mainly because this simplifies the models and our ability to derive quantitative results. However, evidence is gathering from a broad set of observational data that the medium is clumpy at some scale.

The $\mathrm{SiO}, \mathrm{H}_{2} \mathrm{O}$, and $\mathrm{OH}$ maser maps of the inner regions consist of many, often unresolved, brightness spots, but it is essentially impossible to relate this to the small-scale density distribution, since velocity coherence along the line of sight plays, exponential amplification may play, and in the case of $\mathrm{OH}$ photodissociation plays, a major role. VLBI observations of $\mathrm{SiO}$ masers suggest spot sizes as small as a few $10^{12} \mathrm{~cm}$ (Colomer et al. 1992). Assuming a clump size of $10^{13} \mathrm{~cm}$ and $\mathrm{n}_{\mathrm{H}_{2}} \approx 10^{10} \mathrm{~cm}^{-3}$ (the SiO masers are quenched at higher densities) leads to a clump mass of $\sim 10^{-7} M_{\odot}$. In fact, for reasonable mass loss rates the gas within $10^{14} \mathrm{~cm}$ of the star must be substantially clumped to provide the densities required for $\mathrm{SiO}$ masers. Measured spot sizes for the $\mathrm{H}_{2} \mathrm{O}$ masers fall in the range $(0.5-2) \times 10^{13}$ $\mathrm{cm}$ at a typical distance of $5 \times 10^{14} \mathrm{~cm}$ from the star (Spencer et al. 1979), while the characteristic $\mathrm{OH}$ maser spot size is a few $10^{14} \mathrm{~cm}$ at a distance of a few $10^{15} \mathrm{~cm}$ from U Ori and U Her as measured in the detailed studies of Chapman et al. (1991, 1994).

The $\mathrm{OH}(1612 \mathrm{MHz})$ masers outline shells that are not uniformly filled with emission. Most of the spatially resolved brightness distributions towards IRC +10216 show a very patchy structure, and in many cases species with quite different excitation characteristics peak in the same region suggesting variations in column density rather than excitation (Guélin et al. 1997). The $\mathrm{CN}(N=1 \rightarrow 0)$ emissions towards U Cam (Lindqvist et al. 1996) and CIT 6 (Lindqvist et al. 1998) are very patchy, and even though CN is a photodissociation product of HCN and its emission may give an enhanced view of any density contrast, it is hard to reconcile these data with a smooth medium.

The $\mathrm{CO}$ emission is probably better related to the true density distribution than are any other molecular emissions. Therefore, the large, geometrically thin CO shells around the C stars U Ant, S Sct, and TT Cyg are probably the best objects for a study of the small-scale structure since there is very little circumstellar material along the line of sight, i.e. effects due to saturation or clump overlap are limited (Olofsson et al. 1996). The brightness maps have a clear patchy appearance ( $10-20$ major clumps), but it has been shown that to infer the small-scale density distribution from these maps is not so easy. Emission from about $10^{3}$ identical, unresolved clumps that are randomly positioned within the shell gives a large-scale 
brightness distribution with a patchy appearance, at the scale set by the observational resolution, that resembles the observed ones (Bergman et al. 1993; Olofsson et al. 1996). Recent interferometer observations of a part of the shell of S Sct at an angular resolution of $\sim 5^{\prime \prime}$ show many unresolved clumps, and an extrapolation to the entire shell gives $\sim 500$ clumps (Bergman et al. 1998).

There is also evidence for clumpiness from the observations of post-AGB objects. In general, the patchiness of the $\mathrm{CO}$ brightness maps increases with the evolutionary stage of the central object (Bachiller et al. 1993). The most extreme example of this is the direct observation of $\mathrm{CO}$ emission from cometary globules in the Helix nebula (Huggins et al. 1992). The estimated clump properties are: $M=5 \times 10^{-6} M_{\odot}, n_{\mathrm{H}_{2}} \leq 10^{5} \mathrm{~cm}^{-3}, T$ $=25 \mathrm{~K}$. Finally, several molecular species show anomalously strong lines in the more evolved objects, e.g. $\mathrm{HCN}, \mathrm{CN}$, and $\mathrm{HCO}^{+}$, indicating that a photo-induced chemistry is initiated in high-density clumps (Deguchi et al. 1992; Cox et al. 1992; Howe et al. 1994).

\section{A Scenario}

In this final section we will try to give a possible explanation for the apparent morphology change of CSEs with evolutionary stage. We adopt the (perhaps somewhat extreme) view point that the medium is highly clumped already at its ejection from the central star. As a typical example, we suppose that one clump of mass $10^{-6} M_{\odot}$ is ejected per year (i.e., rougly one per pulsational period), i.e. $\dot{M}=10^{-6} M_{\odot} \mathrm{yr}^{-1}$. The clump evolution as it recedes from the star is determined by the thermal motion, which in turn is determined by heating and cooling processes. We will follow the parametric treatment of this evolution that was introduced in Bergman et al. (1993), and also adopt the same parameters, which were shown by Olofsson et al. (1996) to give a reasonable explanation for the CO shells around the C stars R Scl, U Ant, S Sct, and TT Cyg (mainly the ages of the shells were changed). With an expansion velocity of $10 \mathrm{~km} \mathrm{~s}^{-1}$ we find: within $3 \times 10^{14} \mathrm{~cm}$ there are 10 clumps and $n_{\mathrm{H}_{2}}>10^{9} \mathrm{~cm}^{-3}$, i.e. this is the regime of the $\mathrm{SiO}$ masers, and the $\mathrm{H}_{2} \mathrm{O}$ masers are quenched above this density; within $3 \times 10^{15} \mathrm{~cm}$ there are 100 clumps and $n_{\mathrm{H}_{2}}>4 \times 10^{6} \mathrm{~cm}^{-3}$, i.e. the $\mathrm{OH}$ masers are found at the edge of this region (where $\mathrm{OH}$ is formed from the photodissociation of $\mathrm{H}_{2} \mathrm{O}$ ); and within $3 \times 10^{17} \mathrm{~cm}$ there are $10^{4}$ clumps and $n_{\mathrm{H}_{2}}>10^{2} \mathrm{~cm}^{-3}$, i.e. at the outer edge $\mathrm{CO}$ is barely excited and it is, in fact, photodissociated before reaching this radius. Clump blocking along the line of sight occurs already at $\sim 2 \times 10^{15} \mathrm{~cm}$. If one takes into account that the velocity dispersion within the clumps is much smaller than the expansion velocity, the blocking (in a narrow velocity range) occurs at much 
larger radii. The transition to a smooth medium occurs first at $\sim 10^{19} \mathrm{~cm}$.

In this scenario the $\mathrm{SiO}$ masers are located inside the CSE as expected from theory (the $\mathrm{SiO}$ abundance is too low once dust starts to form), and as indicated by observations (see, e.g., Greenhill et al. 1995). In the region where the $\mathrm{H}_{2} \mathrm{O}$ masers are located there are too few clumps to give any reliable information on the morphology. The same applies partly to the $\mathrm{OH}(1665 / 1667 \mathrm{MHz})$ maser region for low $\dot{M}$, while for higher $\dot{M}$ accidental overlap of clumps combined with unsaturated amplification may give a highly perturbed view of the geometry. In the regions where the $\mathrm{OH}(1612 \mathrm{MHz})$ masers (for higher $\dot{M})$ and the CO line-emitting gas are located, the clump overlap is substantial and the brightness contrast is consequently decreased. One should also note that a clumpy medium leads to circular brightness distributions (if $v_{\mathrm{e}}$ is isotropic) because the photodissociation, which normally limits the brightness distribution, is determined by the clump properties and not $\dot{M}(\theta, \varphi)$. This, combined with the geometrical projection effect, leads to brightness distributions from which an overall spherical symmetry is inferred, even though moderate equatorial density enhancements may be present. Any asymmetry, if existing, is most easily seen at small scales, and in the case of emission from geometrically thin shell regions.

Another interesting property of a clumpy medium is that since the photodissociation is determined by the clump properties, we would expect the sizes of molecular envelopes to be less dependent on the mass-loss rate than derived from "smooth density" models (unless the clump properties strongly depend on the mass-loss rate). Also the cut-offs in the molecular distributions will be much smoother than otherwise expected, and regions where the chemistry has developed differently will become mixed.

During the early post-AGB evolution an essentially isotropic, highvelocity, smooth wind starts to blow. Even if the equatorial density enhancement of the AGB-CSE is only moderate, the clumps in the polar region will be preferentially accelerated to higher velocities, while the clumps in the equatorial region start to pile up (in these clumps a completely different shock-induced chemistry may develop). A cavity develops, and the clumps at lower latitudes start to accelerate to intermediate velocities, while the material in the polar region gradually disappears. In this way an elliptical shell or an hour-glass morphology develops, depending on the strength of the equatorial density enhancement in the original AGB-CSE. At this point the UV-flux from the central star may drastically enhance the contrast in the brightness distribution as a result of increased photodissociation in directions of low optical depth (this may also lead to a new photo-induced chemistry). Finally, only the equatorial density enhancement remains, and the medium is very clumpy. 
I am grateful to the Swedish Natural Sciences Research Council (NFR) and the IAU for travel support.

\section{References}

Alcolea, J. \& Bujarrabal, V. 1995, $A \& A$, 303, L21

Alcolea, J., Bujarrabal, V. \& Sánchez Contreras, C. 1996, $A \& A, 312,560$

Bachiller, R., Bujarrabal, V., Martin-Pintado, J.\& Gómez-González, J. 1989a, $A \& A, 218$, 252

Bachiller, R., Huggins, P. J., Cox, P. \& Forveille, T. 1991, $A \& A, 247,525$

Bachiller, R., Huggins, P. J., Cox, P. \& Forveille, T. 1993, A\&A, 267, 177

Bachiller, R., Planesas, P., Martin-Pintado, J., Bujarrabal, V. \& Tafalla, M. 1989b, $A \& A$, 210,366

Benson, P. J., Little-Marenin, I. R., Woods, T. C., et al. 1990, ApJ Supp., 74, 911

Bergman, P., Carlström, U. \& Olofsson, H. 1993, A\&A, 268, 685

Bergman, P., Olofsson, H. \& Bieging, J. H. 1998, in prep.

Bieging, J. H. \& Nguyen-Q-Rieu 1996, $A J, 112,706$

Bieging, J.H. \& Tafalla, M. 1993, $A J, 105,576$

Bieging, J. H., Wilner, D. \& Thronson, H. A. Jr. 1991, ApJ, 379, 271

Bowers, P.F. \& Johnston, K. J. 1990, ApJ, 354, 676

Bowers, P.F. \& Johnston, K. J. 1994, ApJ Supp., 92, 189

Bowers, P.F., Johnston, K. J. \& de Vegt, C. 1989, ApJ, 340, 479

Bujarrabal, V. \& Alcolea, J. 1991, $A \& A, 251,536$

Bujarrabal, V., Alcolea, J., Neri, R. \& Grewing, M. 1994, ApJ, 436, L169

Carlstrom, J. E., Welch, W. J., Goldsmith, P. F. \& Lis, D. C. 1990, $A J, 100,213$

Cernicharo, J., Brunswig, W., Pauber, G. \& Liechti, S. 1994, ApJ, 423, L143

Cernicharo, J., Guélin, M., Martin-Pintado, J., Peñalver, J. \& Mauersberger, R. 1989, $A \& A, 222, \mathrm{~L} 1$

Chapman, J. M., Cohen, R. J. \& Saikia, D. J. 1991, MNRAS, 249, 227

Chapman, J. M., Sivagnanam, P., Cohen, R. J. \& Le Squeren, A. M. 1994, MNRAS, 268, 475

Cherchneff, I. \& Glassgold, A.E. 1993, $A p J, 419$, L41

Colomer, F., Baudry, A., Graham, D. A., et al. 1996, $A \& A, 312,950$

Colomer, F., Graham, D. A., Krichbaum, T. P., et al. 1992, $A \& A$, 254, L17

Cox, P., Omont, A., Huggins, P. J., Bachiller, R. \& Forveille, T. 1992, $A \& A, 266,420$

Dayal, A. \& Bieging, J.H. 1995, ApJ, 439, 996

Deguchi, S., Izumiura, H., Nguyen-Q-Rieu, Shibata, K. M., Ukita, N. \& Yamamura, I. $1992, A p J, 392,597$

Diamond, P.J., Kemball, A.J., Junor, W., Zensus, A., Benson, J. \& Dhawan, V. 1994, $A p J, 430, \mathrm{~L} 61$

Gammie, C. F., Knapp, G. R., Young, K., Phillips, T. G. \& Falgarone, E. 1989, ApJ, 345, L87

Gensheimer, P. D., Likkel, L. \& Snyder, L. E. 1995, ApJ, 439, 445

Graham, J. R., Serabyn, E., Herbst, T. M., et al. 1993, $A J, 105,250$

Greenhill, L. J., Colomer, F., Moran, J. M., Backer, D. C., Danchi, W. C. \& Bester, M. $1995, A p J, 449,365$

Guélin, M., Lucas, R. \& Cernicharo, J. 1993, $A \& A, 280$, L19

Guélin, M., Lucas, R. \& Neri, R. 1997, in IAU Symp. 170: CO: Twenty-Five Years of Millimeter-Wave Spectroscopy, ed. W. B. Latter et al. (Kluwer), p. 359

Hajian, A. R., Phillips, J. A. \& Terzian, Y. 1995 ApJ, 446, 244

Herman, J., Baud, B., Habing, H. J. \& Winnberg, A. 1985, $A \& A, 143,122$

Howe, D. A., Hartquist, T.W. \& Williams, D. A. 1994, MNRAS, 271, 811

Huggins, P. J., Bachiller, R., Cox, P. \& Forveille, T. 1992, ApJ, 401, L43

Huggins, P. J., Bachiller, R., Cox, P. \& Forveille, T. 1996, $A \& A, 315,284$ 
Jaminet, P. A., Danchi, W. C., Sandell, G. \& Sutton, E. C. 1992, ApJ, 400, 535

Jaminet, P. A., Danchi, W.C., Sutton, E. C., et al. 1991, ApJ, 380, 461

Jura, M., Balm, S. P. \& Kahane, C. 1995, ApJ, 453, 721

Kahane, C., Audinos, P., Barnbaum, C. \& Morris, M. 1996, $A \& A, 314,871$

Kahane, C. \& Jura, M. 1996, $A \& A, 310,952$

Knapp, G. R., Jorissen, A. \& Young, K. 1997, $A \& A, 326,318$

Lindqvist, M., Lucas, R., Olofsson, H., Omont, A., Eriksson, K.\& Gustafsson, B. 1996, $A \& A, 305, \mathrm{~L} 57$

Lindqvist, M., Lucas, R., Olofsson, H., et al. 1998, in prep.

Loup, C., Forveille, T., Omont, A. \& Paul, J. F. 1993, A\&A Supp., 99, 291

Lucas, R., Bujarrabal, V., Guilloteau, S., et al., 1992, $A \& A, 262,491$

Lucas, R., Guélin, M., Kahane, C., Audinos, P. \& Cernicharo, J. 1995, in Circumstellar Matter 1994, ed. G. D. Watt and P. M. Williams (Kluwer), p. 293

Lucas, R. \& Guilloteau, S. 1992, A\&A, 259, L23

Martin-Pintado, J., Gaume, R. A., Johnston, K. J. \& Bachiller, R. 1995, ApJ, 446, 687

Miyoshi, M., Matsumoto, K., Kameno, S., Takaba, H. \& Iwata, T. 1994, Nature, 371, 395

Millar, T. J. \& Herbst, E. 1994, $A \& A, 288,561$

Nercessian, E., Guilloteau, S., Omont, A. \& Benayoun, J. J. 1989, A\&A, 210, 225

Neri, R., García-Burillo, S., Guélin, M., Cernicharo, J., Guilloteau, S. \& Lucas, R. 1992, $A \mathscr{E} A, 262,544$

Nguyen-Q-Rieu, Winnberg, A. \& Bujarrabal, V. 1986, $A \& A, 165,204$

Nyman, L.- $\AA$., Olofsson, H., Rogers, C., Heske, A. \& Sahai, R. 1993, in Mass Loss on the $A G B$ and Beyond, ed. H. E. Schwarz, ESO Conference and Workshop Proceedings No. 46 , p. 451

Olofsson, H. 1989, in IAU Coll. 106: Evolution of Peculiar Red Giant Stars, ed. H.R. Johnson and B. Zuckerman (Cambridge Univ. Press), p. 321

Olofsson, H. 1997, in IAU Symp. 178: Molecules in Astrophysics: Probes and Processes, ed. E. F. van Dishoeck (Kluwer), p. 457

Olofsson, H., Bergman, P., Eriksson, K. \& Gustafsson, B. 1996, $A \& A, 311,587$

Phillips, J. P., Williams, P. G., Mampaso, A. \& Ukita, N. 1992, $A \& A, 260,283$

Planesas, P., Kenney, J. D. P. \& Bachiller, R. 1990, ApJ, 364, L9

Reid, M. J. \& Menten, K. M. 1990, ApJ, 360, L51

Sahai, R. 1990, ApJ, 362, 652

Sahai, R. 1992, $A \& A, 253$, L33

Sahai, R. \& Bieging, J. H. 1993, AJ, 105, 595

Sahai, R., Wootten, A., Schwarz, H.E. \& Clegg, R. E.S. 1991, $A \& A, 251,560$

Sahai, R., Wootten, A., Schwarz, H.E. \& Wild, W. 1994, ApJ, 428, 237

Spencer, J. H., Johnston, K. J., Moran, J. M., Reid, M. J. \& Walker, R. C. 1979, ApJ, 230, 449

Stanek, K.Z., Knapp, G. R., Young, K. \& Phillips, T. G. 1995, ApJ Supp., 100, 169

Takano, S., Saito, S. \& Tsuji, T. 1992, PASJ, 44, 469

Truong-Bach, Morris, D. \& Nguyen-Q-Rieu 1991, $A \& A, 249,435$

Truong-Bach, Morris, D., Nguyen-Q-Rieu \& Deguchi, S. 1990, $A \& A, 230,431$

van Loon, J. Th., Zijlstra, A. A., Bujarrabal, V. \& Nyman, L.- $\AA .1996, A छ A, 306$, L29

Welty, A. D., Fix, J.D. \& Mutel, R. L. 1987, $A p J, 318,852$

Wood, P. R., Whiteoak, J.B., Hughes, S.M.G., Bessell, M.S., Gardner, F. \& Hyland, A. R. $1992, A p J, 397,552$

Yamamura, I., Onaka, T., Kamijo, F., Deguchi, S. \& Ukita, N. 1995, ApJ, 439, L13

Yamamura, I., Onaka, T., Kamijo, F., Deguchi, S. \& Ukita, N. 1996, ApJ, 465, 926

Yates, J. A. \& Cohen, R. J. 1994, MNRAS, 270, 958

Young, K., Serabyn, G., Phillips, T.G., Knapp, G. R., Güsten, R. \& Schulz, A. 1992, ApJ, 385, 265 


\section{Discussion}

Elitzur: Two comments: (1) SiO comes from inside the dust formation zone. Models that attempt to integrate the atmosphere and the wind should consider the $\mathrm{SiO}$ observations. Polarization shows that the magnetic field is a significant dynamic force. (2) Excitation may make a symmetric shell look asymmetrical and vice versa. In IRC +10216, the IR images are asymmetric at short wavelengths and spherically symmetric at long wavelengths.

Olofsson: I agree with both comments. Concerning the excitation, one has to use many transitions and many molecular species before one can draw any definite conclusions about the large-scale density structure.

Whitelock: I am worried about the effects of duplicity in the general interpretation of evolution from the AGB to PNe. Some of the stars you describe as early post-AGB are interacting binaries. Hans Van Winckel provided us with a clear indication that the so-called "post-AGB stars" with a near-IR excess are binaries which could not have undergone normal AGB evolution (their binary periods are too short). So I would like to make a general request that we stop calling these stars "post-AGB."

Olofsson: I agree that there are objects that should not be included in this discussion, and I have tried to avoid them. However, binarity is probably also common for true AGB and post-AGB objects, and it will probably have an effect on the mass-loss geometry on the AGB, in particular the strength of the equatorial density enhancement. This, in turn, will have an effect on the morphology of, for instance, planetary nebulae formed by the interaction of a fast wind and the slow AGB wind.

Richards: Just like your last model, bipolar outflow on a very small scale is seen in $\mathrm{H}_{2} \mathrm{O}$ masers from NML Cyg. Monitoring over years shows what clumps survive proper motion in the NML Cyg outflow, and random effects in more symmetric shells. Individual clumps are well resolved in $\mathrm{H}_{2} \mathrm{O}$ maser emission. So far supergiants have been analyzed; Miras will also be mapped. 